\title{
Twinning stress prediction in bec metals and alloys
}

\author{
Avinesh Ojha and Huseyin Sehitoglu* \\ Department of Mechanical Science and Engineering, University of Illinois, 1206 West Green \\ Street, Urbana, IL 61801, USA
}

(Received 20 June 2014; accepted 11 August 2014)

\begin{abstract}
The development of a twin stress relationship for bcc metals and alloys in agreement with experiments has been both scientifically challenging and technologically vital. A modified approach to Peierls-Nabarro model is formulated that predicts the twinning stress in excellent agreement with experiments. We utilize the first principles energy calculations to extract the energy landscape associated with twinning and obtain the disregistry function to account for the interaction of multiple dislocations comprising the twin. The metals and alloys considered include $\mathrm{Fe}, \mathrm{V}, \mathrm{Nb}, \mathrm{Ta}, \mathrm{Mo}, \mathrm{W}, \mathrm{Fe}-3$ at.\%V, $\mathrm{Fe}-35$ at.\%Ni and $\mathrm{Fe}-3$ at.\%Si. The variation of twinning stress within metals is substantial (90-800 MPa) and depends primarily on the twin boundary migration energy, the shear moduli, the interplanar spacing and the geometrical positions of the fractional dislocations constituting the twin.
\end{abstract}

Keywords: twinning; density-functional theory; Fe-based alloys

\section{Introduction}

The mechanism of twin nucleation in bcc crystals, with differing dislocation arrangements, has been studied for the past several decades [1,2]. The detailed microscopic observations in bcc crystals support the fact that a pre-existing dislocation configuration is a prerequisite to twin nucleation [3,4]. The most widely accepted mechanism suggests a twin to nucleate by the dissociation of a perfect $1 / 2\left\langle\begin{array}{lll}1 & 1 & 1\end{array}\right)$ screw dislocation into three $1 / 6\left\langle\begin{array}{lll}1 & 1 & 1\end{array}\right\rangle$ fractionals, and their subsequent arrangement on $\left\{\begin{array}{lll}1 & 1 & 2\end{array}\right\}$ plane [5]. During the twin nucleation process, the dislocations have to overcome successive energy barriers represented by the Generalized Planar Fault Energy (GPFE) curve. The GPFE curve is obtained as the energy change per fault area to create successive twin layers. Several approaches have been undertaken to predict the twinning stress for bcc metals in the past $[3,4,6-10]$; however, the twinning stress expressions introduce a number of empirical constants that are difficult to obtain, or predict high stress compared to the experimental values. The earliest twinning stress model was proposed by Cottrell and Bilby [8] based on the pole mechanism. The twinning stress expression for pole mechanism is given by $\tau=\frac{\gamma_{u s}}{b_{p}}$, where $\gamma_{u s}$ is the unstable stacking fault energy and $b_{p}$ is the twinning dislocation. Based on this formulation, the estimated twinning stress for $\mathrm{Fe}$ is $1050 \mathrm{MPa}$ which is at least five times higher than experimental value of $180 \mathrm{MPa}$. Sleeswyk's twinning stress formula $\left(\tau=\frac{\gamma_{s f}}{3 b_{p}}\right)$ is based on the dissociation of

\footnotetext{
*Corresponding author. Email: huseyin@illinois.edu
} 
a $1 / 2\left\langle\begin{array}{lll}1 & 1 & 1\end{array}\right\rangle$ screw dislocation into three $1 / 6\left\langle\begin{array}{lll}1 & 1 & 1\rangle\end{array}\right\rangle$ twinning fractionals [3]. Using this expression, the twinning stress for Fe is estimated as $250 \mathrm{MPa}$. Similarly, the twinning mechanism based on the dissociation of an $1 / 2\left\langle\begin{array}{lll}1 & 1 & 1\end{array}\right)$ edge dislocation has been proposed [9], and the twin nucleation stress expression is written as $\tau=k_{t} \frac{\mu}{2 \pi(1-v)}$, where $\mu$ is the shear modulus, $v$ is the Poisson's ratio and $k_{t}$ is an experimental constant. The calculation based on the edge dislocation dissociation predicts the twinning stress for $\mathrm{Fe}$ as $430 \mathrm{MPa}$. Finally, the theoretical twinning stress [11] which is calculated from the maximum slope of the GPFE curve $\left(\tau=\frac{\pi \gamma_{T B M}}{b_{p}}=1200 \mathrm{MPa}\right)$ tends to be an order of magnitude higher than the experimental value. We point out that the estimation of such high twinning stress is due to (i) the incomplete description of the energetics associated with twinning and (ii) the insufficient geometrical consideration of the fractional dislocations. In the present paper, we address these issues related to the assessment of such high twinning stress, and building upon our recent work, propose a modified Peierls-Nabarro (PN) formalism to predict the twinning stress accurately in technologically important bec metals and alloys.

Several authors have modified the original PN model to compute the core properties and the 'Peierls stress for slip' in cubic and hcp metals [12-24], but the model has not been extended to account for the twinning process in bcc metals. In the present work, we redefine the energetics of the twinning process and the dislocation configuration within the framework of PN model [25] and predict the twinning stress in pure metals (Fe, $\mathrm{V}, \mathrm{Nb}$, Ta, Mo and $\mathrm{W}$ ) and alloys (Fe-25\%Ni, Fe-3\%V and $\mathrm{Fe}-3 \% \mathrm{Si}$ ). To do so, we make several modifications to the original PN formalism. We incorporate the energy barriers (GPFE) associated with the twin nucleation process as a function of the positions of the dislocations (disregistry) and incorporate it into the discrete form of the misfit energy integral. Overall, the modified PN formalism in the present work captures the essential energetics of the twinning process in bec metals and predicts the twinning stress in better agreement with the experimentally measured values compared to other models.

We note that in the original PN formulation, the energy variation with respect to the disregistry of a dislocation is obtained from Frenkel expression consisting of a single sinusoidal term [26]. However, in actual crystals, the energy landscape is quite complex. Foreman et al. [27] first considered the effect of such functional form on the effect on Peierls stress, and recent studies considered atomistic calculations in an attempt to establish the form of the landscape [26,28]. One of the major advancements of the current analysis is estimating the misfit energy associated with twinning dislocations from the GPFE at the local disregistry, and calculating the twinning stress. The misfit energy across a glide plane is defined as the sum of misfit energies between pairs of atomic planes and can be obtained from the GPFE as follows [28].

$$
E_{\text {misfit }}=\int_{-\infty}^{+\infty} \gamma_{G P F E}(f(x))
$$

where $f(x)$ is the disregistry function in terms of the dislocation position $x$, and represents the inelastic displacements across the glide plane of the dislocation. The solution to the disregistry function $f(x)$ for a single dislocation has been obtained [25,26]. In the present case, we modify the disregistry function $f(x)$ to capture the displacements associated with the twinning dislocations to accurately obtain the misfit energy. The GPFE 
is complicated in its form, and we use a series of sinusoidal terms to represent it. Due to the form of the GPFE, no analytical solution of the Peierls stress can be obtained for twinning, and we use the numerical approach to estimate the stress. We plot the results in normalized form (shown later), so the twinning stress magnitudes show the dependence on the most important variables.

Following the early works of Vítek [29] and Joos et al. [15], we modify the misfit energy integral to account for the variation of the disregistry as the twinning dislocation cores move infinitesimally from one position to the other along the interface. With such a modification in the misfit energy term, we capture the energy distribution as a function of the position of the dislocation cores of the twin nucleus [24]. Thus, the discrete form of the misfit energy can be written as follows [26].

$$
E_{G P F E}=\sum_{-\infty}^{+\infty} \gamma_{t w i n}\left[f\left(m a^{\prime}-u\right)\right] a^{\prime}
$$

where $m$ is an integer, $u$ is the position of the dislocation line and $a^{\prime}$ is the lattice periodicity defined as the shortest distance between two equivalent atomic rows in the direction of the dislocation displacement $[11,12,14,16,20]$. The term $\gamma_{\text {twin }}\left[f\left(m a^{\prime}-u\right)\right]$ is obtained from the GPFE similar to Wang et al. [30, 31]. We note that the disregistry function $f\left(m a^{\prime}-u\right)$ and hence, the misfit energy expression depends on the positions of the fractional dislocations as follows:

$$
f(x)=\frac{b}{N \pi}\left\{\tan ^{-1}\left(\frac{x}{\xi}\right)+\tan ^{-1}\left(\frac{x-d_{1}}{\xi}\right)+\tan ^{-1}\left(\frac{x-\left(d_{1}+d_{2}\right)}{\xi}\right)\right\}+\frac{b}{2}
$$

where $N$ corresponds to the number of twinning dislocations in the present analysis, $\xi$ represent the half-core width of the dislocation, and $d_{1}$ and $d_{2}$ represents the positions of the fractional dislocations of the twin nucleus. In the present work, we obtain the total energy of the twinning dislocations by incorporating the elastic interactions of the dislocations at the mesoscale with the misfit energy obtained from the density functional theory (DFT) calculations. We minimize the total energy with respect to the separation distances $\left(d_{1}\right.$ and $d_{2}$ ) of the twinning fractionals and obtain the twinning stress for bec crystals.

We consider that a twin nucleates by the rearrangement of the fractional dislocations of a dissociated $1 / 2\left\langle\begin{array}{lll}1 & 1 & 1\end{array}\right\rangle$ screw dislocation [3]. Atomistic calculations [32] have shown that the $1 / 2\left\langle\begin{array}{lll}1 & 1 & 1\end{array}\right\rangle$-screw dislocation in a bcc core can be considered as the generalized splitting of the screw dislocation into three fractional dislocations (Figure 1a) as follows:

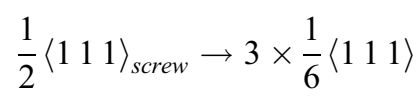

The stable energy configuration of the three-layer twin nucleus has been validated by the simulation results [33] and the experimental observations [5,34]. The three fractional dislocations possess a three-fold configuration (see Figure 1a) symmetric to the $\left\langle\begin{array}{lll}1 & 1 & 1\end{array}\right\rangle$ screw axis. Based on the theoretical calculations [4], the width $(w)$ of the $a / 2\left[\begin{array}{lll}1 & 1 & 1\end{array}\right]$ dissociated core in a bcc crystal is predicted to be $1 b-2 b$ where $b=\frac{\sqrt{3} a}{2}$. The separation distance $(x)$ between any pair of fractional dislocations in the bcc metals considered, therefore, varies from $2 d_{\left\{\begin{array}{lll}1 & 1 & 2\end{array}\right\}}$ to $4 d_{\left\{\begin{array}{lll}1 & 1 & 2\end{array}\right\}}$, where $d_{\left\{\begin{array}{lll}1 & 1 & 2\end{array}\right\}}$ is the $\left\{\begin{array}{lll}1 & 1 & 2\end{array}\right\}$ interplanar 

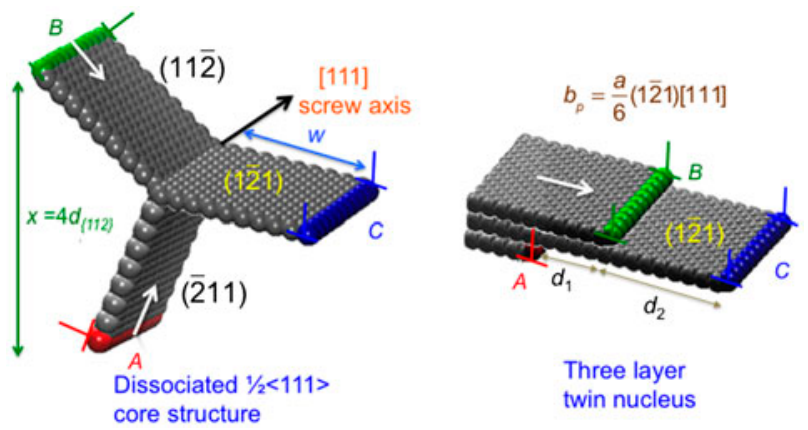

Figure 1. (a) The symmetric configuration of the three fractional dislocations in a bcc crystal formed as a result of the dissociation of the $1 / 2\left\langle\begin{array}{lll}1 & 1 & 1\end{array}\right)$ screw dislocation on three $\left\{\begin{array}{llll}1 & 1 & 2\end{array}\right\}$ planes. (b) Under applied stress, the dislocations $A$ and $B$ translate to the most stressed $\left\{\begin{array}{lll}1 & 1 & 2\end{array}\right\}$ plane to form a three-layer twin nucleus. The figure is not drawn to scale. Thickening of the twin occurs by the motion of the additional fractional dislocations over the existing twin boundary [3].

distance. Under applied stress, the dislocations $A$ and $B$ translate along their individual planes $\left(\left(\begin{array}{lll}2 & 1 & 1\end{array}\right)\right.$ and $\left.\left(\begin{array}{lll}1 & 1 & \overline{2}\end{array}\right)\right)$ and later cross-slip onto the most stressed $\left(\begin{array}{lll}1 & \overline{2} & 1\end{array}\right)$ plane to form a twin as shown in Figure 1a and b. During this process, the dislocations have to overcome the Peierls energy barrier or the GPFE.

The generalized gradient approximation was used to the density functional theory using the Vienna-ab initio simulation package [35,36]. Periodic boundary conditions were imposed across the supercell to represent the bulk material. The $k$-point meshes for Brillouin zone sampling were constructed using the Monkhorst-Pack scheme. A plane wave cut-off energy of $500 \mathrm{eV}$ and an $9 \times 9 \times 9 k$-point mesh for the unit cell were found to be sufficient to converge the total energy to better than $10^{-5} \mathrm{~V} /$ atom. Table 1 shows the lattice constants, and the $\left\{\begin{array}{lll}1 & 1 & 2\end{array}\right\}\left\langle\begin{array}{lll}1 & 1 & 1\end{array}\right\rangle$ shear moduli obtained for the various bcc metals and alloys, and comparison with other experimental or DFT calculations. We will provide the other parameters during the course of the paper.

Table 1. Comparison of the lattice constants (in $\AA$ ) and the shear moduli $\left(\mu_{\{112\}\langle 111\rangle}\right.$ in GPa) for the bcc metals and alloys considered in the present study with the experimental values or other DFT calculations.

\begin{tabular}{lccccc}
\hline & \multicolumn{2}{c}{ Lattice constants $(\AA)$} & & \multicolumn{2}{c}{$\mu_{\{112\}\langle 111\rangle}(\mathrm{GPa})$} \\
\cline { 2 - 3 } \cline { 5 - 6 } Material & DFT (this study) & Experimental/DFT* & & DFT (this study) & Experimental/DFT* \\
\hline $\mathrm{Fe}$ & 2.851 & $2.86[37]$ & & 40 & $40[38]$ \\
$\mathrm{V}$ & 3.002 & $3.038[39]$ & & 25 & $27[40,41]$ \\
$\mathrm{Nb}$ & 3.310 & $3.294[42]$ & & 23 & $20[40,41]$ \\
$\mathrm{Ta}$ & 3.265 & $3.33[43]$ & & 32 & $35[40,41]$ \\
$\mathrm{Mo}$ & 3.130 & $3.147[44]$ & & 100 & $92[45]$ \\
$\mathrm{W}$ & 3.150 & $3.155[46]$ & & 109 & $115[47]$ \\
$\mathrm{Fe}-3$ 3at.\%Si & 2.855 & $2.86[48]^{*}$ & & 56 & $58[48]^{*}$ \\
$\mathrm{Fe}-3 a t . \% \mathrm{~V}$ & 2.866 & $2.87[48]^{*}$ & & 59 & $57[48]^{*}$ \\
$\mathrm{Fe}-25$ at.\%Ni & 2.882 & - & 31 & - \\
\hline
\end{tabular}

Note: The values marked with $(*)$ are obtained from DFT. 
We used a 25-layered periodic supercell consisting of 66 atoms for $\mathrm{Fe}-3 \mathrm{at} . \% \mathrm{Si}$

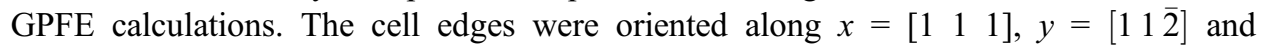
$z=\left[\begin{array}{lll}1 & 1 & 0\end{array}\right]$ directions. We allowed displacement relaxation in the crystal along $\left[\begin{array}{lll}1 & 1 & \overline{2}\end{array}\right]$ direction. This is important to maintain the twin symmetry. An Fe atom was substituted by a $\mathrm{Si}$ atom within the 10th and 12th layers on either sides of the 11th layer fault plane. The first layer stacking fault energy (SFE) value $\left(\gamma_{s f}\right)$ was obtained by rigidly sliding the layers 12 and above with respect to the bottom layers 1-11, the second layer SFE $\left(2 \gamma_{t s f}\right)$ was obtained by sliding layers 13 and above with respect to the layers 1-12 and so on. In such a case, we find that $\mathrm{Si}$ atoms maintain the positional symmetry across the twin boundary after the first layer fault. These positions are found to lead to the lowest energy configuration, and are consistent with the findings for solid-solution fcc alloys as well [49-51]. In fact, when solute atom is adjacent to the fault, the energy of the stable energy is reduced compared to the case where the solute atom is away from the fault (discussed later). Similar atomic configuration was followed for obtaining the GPFE values for $\mathrm{Fe}-3$ at.\% $\mathrm{V}$ and $\mathrm{Fe}-25 \mathrm{at} . \% \mathrm{Ni}$.

The GPFE in bcc crystal provides the energy cost per unit area to create multilayer stacking faults on $\left\{\begin{array}{lll}1 & 1 & 2\end{array}\right\}$ planes [29]. The GPFE curves illustrated in Figure 2 were obtained by consecutively shearing the $\left\{\begin{array}{lll}1 & 1 & 2\end{array}\right\}$ planes by a displacement of $a / 6\left\langle\begin{array}{lll}1 & 1 & 1\end{array}\right\rangle$ where "a" is the lattice constant. The peak energy termed the "unstable stacking fault" corresponds to the shearing of the first layer and represents the energy barrier required to nucleate a slip. Similarly, $\gamma_{s f}$ represents the stable stacking fault energy, $\gamma_{u t}$ represents the energy barrier required to nucleate a twin and $\gamma_{T B M}$ represents the energy barrier required to create additional faults once a stable three-layer twin is formed. Table 2 represents the most comprehensive collection of bcc GPFE information to date. We note that for the bcc metals considered, $\gamma_{T B M}$ does not change once a three-layer twin is nucleated. The procedure for calculating GPFE in case of pure metals is well established; however, the solid solution alloys provide a number of possible energy pathways depending on the positions of the solute atoms. In this case, we seek a particular

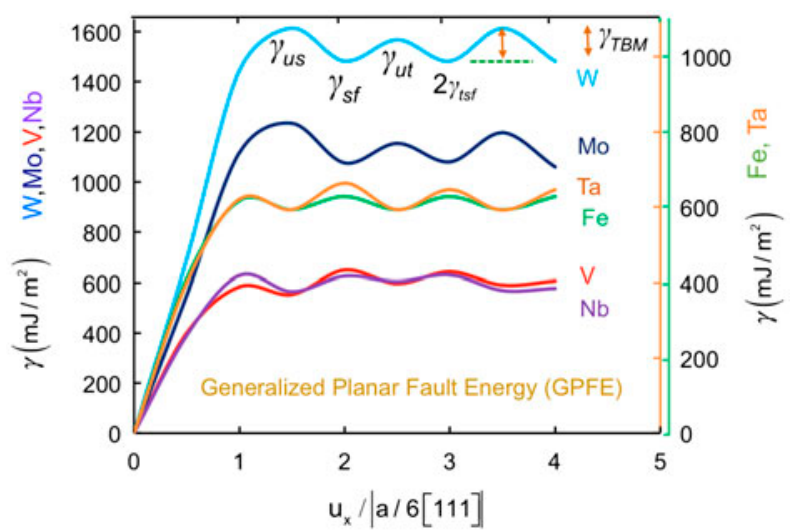

Figure 2. GPFE obtained for the bcc alloys obtained by shearing $n$ consecutive $\left\{\begin{array}{lll}1 & 1 & 2\end{array}\right\}$ layers by a displacement of $a / 6\left\langle\begin{array}{llll}1 & 1 & 1\end{array}\right\rangle$. The energy parameters $\gamma_{s f}, \gamma_{u t}$ and $\gamma_{T B M}$ are marked for $\mathrm{W}$ for clarity of the figure. The values for $\mathrm{Fe}$ and $\mathrm{Ta}$ are read from the right axes while other GPFE curves correspond to the left axis. 
Table 2. The GPFE parameters (in $\mathrm{mJ} \mathrm{m}^{-2}$ ) for the alloys investigated in the present study. The twin boundary migration energy $\gamma_{T B M}$ represents the difference in $\gamma_{u t}$ and $2 \gamma_{t s f}$.

\begin{tabular}{lrrrrr}
\hline Material & \multicolumn{1}{c}{$\gamma_{u s}$} & \multicolumn{1}{c}{$\gamma_{s f}$} & $\gamma_{u t}$ & $2 \gamma_{t s f}$ & $\gamma_{T B M}$ \\
\hline $\mathrm{Fe}$ & 617 & 593 & 628 & 588 & 40 \\
$\mathrm{Mo}$ & 1234 & 1076 & 1154 & 1060 & 94 \\
$\mathrm{~V}$ & 582 & 553 & 642 & 598 & 44 \\
$\mathrm{Nb}$ & 628 & 564 & 625 & 578 & 47 \\
$\mathrm{Ta}$ & 621 & 592 & 645 & 594 & 53 \\
$\mathrm{~W}$ & 1614 & 1482 & 1612 & 1482 & 130 \\
$\mathrm{Fe}-3$ at.\%Si & 595 & 45 & 327 & 241 & 107 \\
$\mathrm{Fe}-25$ at.\%Ni & 525 & 464 & 549 & 454 & 95 \\
Fe-3at.\%V & 615 & 546 & 596 & 557 & 39 \\
\hline
\end{tabular}

configuration of the solute position within the parent crystal which yields a minimum total energy, discussed later.

A twin nucleates when the fractional dislocation $A$ in Figure $1 b$ just starts to move under an applied "twinning stress". We obtain the twinning stress by considering the total energy of the dislocations forming the twin, and minimizing it with respect to $d_{1}$ and $d_{2}$. The total energy of the twin nucleus corresponding to Figure $1 \mathrm{~b}$ can be written as follows:

$$
E_{\text {total }}=E_{\text {int }}+E_{\text {GPFE }}+E_{\text {line }}-W
$$

where $E_{\text {int }}$ represents the interaction energy of the dislocations, $E_{G P F E}$ is the twin boundary energy, $E_{\text {line }}$ is the dislocation line energy and $W$ is the externally applied stress. We substitute each of the energy parameters into Equation (4), and obtain the following total energy expression:

$$
\begin{aligned}
E_{\text {total }}= & -\frac{\mu b^{2}}{2 \pi}\left\{\ln \left(\frac{d_{2}}{r_{o}}\right)+\ln \left(\frac{d_{1}+d_{2}}{r_{o}}\right)+\ln \left(\frac{d_{1}}{r_{o}}\right)\right\}+\sum_{m=-\infty}^{m=+\infty} \gamma_{\text {twin }}\left[f\left(m a^{\prime}-u\right)\right] a^{\prime} \\
& +N \frac{\mu b^{2}}{2(1-v)}\left(1-v \cos ^{2} \theta\right)+\sum_{i=1}^{i=2} \tau s h d_{i}
\end{aligned}
$$

where $h$ is the height of the twin nucleus equal to three times the $\left\{\begin{array}{lll}1 & 1 & 2\end{array}\right\}$ interplanar

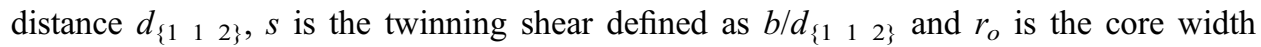

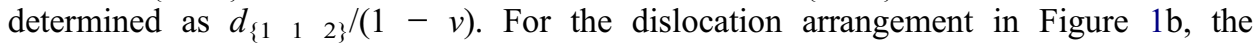
minimum energy configuration is determined by minimizing Equation (5) with respect to $d_{1}$ and $d_{2}$, i.e.

$$
\frac{\partial E_{\text {total }}}{\partial d_{1}}=\frac{\partial E_{\text {total }}}{\partial d_{2}}=0
$$

We numerically solve the set of equations (6) and obtain the critical twinning stress as the minimum value of the applied stress that satisfies these equations.

An inhomogeneous distribution of solute segregated in layers adjacent to the stacking fault (SF) can lead to a minimum stacking fault energy [49,51]. The reason behind the preferential segregation of solute atoms to the planar defects is due to a lower formation energy $\left(\Delta E_{f}\right)$ of solute atoms at the defect compared to the 
substitutional solid solution. In the present work, prior to obtaining the GPFE for bcc alloys, we investigate a number of solute positions that yields a minimum energy configuration. For the case of $\mathrm{Fe}-3$ at. $\% \mathrm{Si}$, we obtained the first-layer stacking fault $\left(\gamma_{s f}\right)$ energy value for a number of Si positions away from the fault, and observed that the energy is the lowest $\left(\gamma_{s f}=45 \mathrm{~mJ} \mathrm{~m}^{-2}\right)$ when the $\mathrm{Si}$ atom is placed adjacent to the fault. In fact, when the position of the $\mathrm{Si}$ atom is on the third layer and away from the fault, the stable stacking fault energy approaches that of the pure $\mathrm{Fe}$ matrix $\left(\gamma_{s f}=593 \mathrm{~mJ} \mathrm{~m}^{-2}\right)$. This is consistent with the Suzuki's extended thermodynamics approach [49] to solute segregation in solid solution. In fact, the solute atoms are either favourable at the stacking fault and lower the stacking fault energy, or may diffuse away from the SF but without increasing the stacking fault energy above the matrix phase. The GPFE for other bcc alloys such as $\mathrm{Fe}-3 \mathrm{at} . \% \mathrm{~V}$ and $\mathrm{Fe}-25 \mathrm{at} \% \mathrm{Ni}$ were obtained in a similar manner. The energy parameters of the GPFE curve for the metals considered in the present analysis are given in Table 2.

We utilize Equations (1)-(3) to compute the misfit energy as a function of the dislocation position. The Peierls stress is the maximum stress required to overcome the periodic barrier, and can be calculated from the maximum slope of the misfit energy as follows [28]:

$$
\tau_{p}=\max \left\{\frac{1}{b} \frac{d E_{\text {misfit }}(u)}{d u}\right\}
$$

We note that the disregistry function in Equation (3) accounts for the multiple dislocation positions comprising the twin, and these positions vary with the bec metal under consideration. We calculated the separation distance $\left(d_{1}\right)$ between the fractional dislocations on the verge of twinning for Fe $(88 \AA)$, Nb $(9 \AA), \mathrm{V}(54 \AA)$, Ta $(2.45 \AA)$, Mo $(0.13 \AA), \mathrm{W}(0.13 \AA), \mathrm{Fe}-25$ at.\%Ni $(1.44 \AA), \mathrm{Fe}-3$ at.\%V $(88 \AA)$ and $\mathrm{Fe}-3 \mathrm{at} . \% \mathrm{Si}$ $(0.32 \AA)$. We observe that the Peierls stress for twinning calculated using our analysis yields much closer results to experiment, and smaller values compared to the theoretical shear strength. For example, the Peierls stress for twinning in Mo using our formulation is $458 \mathrm{MPa}$ in agreement with experiment (472 MPa), and lower than the theoretical stress of $3200 \mathrm{MPa}$.

Figure 3 summarizes the twinning stress for a number of bcc metals and alloys considered in the present analysis as a function of the GPFE parameters, and the separation distance between the fractional dislocations $d_{1}$ and $d_{2}$ (see also Tables 3 and 4). An analysis of Figure 3 reveals that the dependence of twinning stress on the twin boundary migration energy $\gamma_{T B M}\left(=\gamma_{u t}-2 \gamma_{t s f}\right)$ and on $d_{1}$ and $d_{2}$ is substantial. As a first approximation, the increase in $\gamma_{T B M}$ along with an increase in total separation distance $\left(d_{1}+d_{2}\right)$ between the fractional dislocations increases the twinning stress. Nonetheless, other variables such as shear modulus and $\gamma_{s f}$ also determine the twinning stress. For example, in the case of $\mathrm{Fe}-3 \mathrm{at} . \% \mathrm{Si}$, the total separation distance of $76 \AA$ is much lower compared to all other bcc crystals considered, yet the twinning stress of $298 \mathrm{MPa}$ is higher than that of $\mathrm{Fe}, \mathrm{V}, \mathrm{Nb}, \mathrm{Ta}$ and $\mathrm{Fe}-3 \mathrm{at} . \% \mathrm{~V}$. This is due to the higher shear modulus of Fe-3at.\%Si compared to these metals (see Table 1), and a considerably lower $2 \gamma_{t s f}\left(241 \mathrm{~mJ} \mathrm{~m}^{-2}\right)$. The reason behind this strong dependence of the twinning stress on $\gamma_{T B M}$ can be explained based on the energy pathway for twinning. The energy contribution due to the twin nucleation barrier $\gamma_{u t}$ is $2 \gamma_{t s f}+\gamma_{T B M}$ [11]. We know that the formation of twin requires the creation of a stable three-layer fault, and once a 


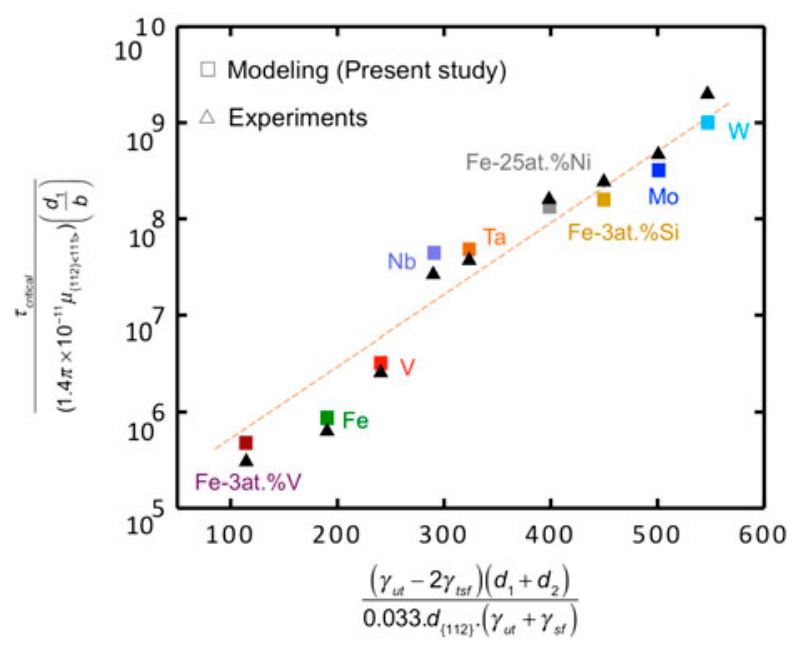

Figure 3. Twinning stress as a function of the GPFE parameters and the positions of the twinning fractionals $d_{1}$ and $d_{2}$. Dashed lines are shown to guide the eye.

Table 3. Comparison of twinning stresses obtained in the present study with the ideal and the experimental values. Present model yields twinning stresses very close to the experimental values.

\begin{tabular}{lccc}
\hline Metals (bcc) & $\begin{array}{c}\tau_{\text {critical }}^{\text {exp }}(\mathrm{MPa}) \\
(\text { experimental) }\end{array}$ & $\begin{array}{c}\tau_{\text {critical }}^{\text {ideal }}(\mathrm{MPa}) \\
\text { (theory) }\end{array}$ & $\begin{array}{c}\tau_{\text {critical }}^{\text {current }}(\mathrm{MPa}) \\
\text { (present model) }\end{array}$ \\
\hline $\mathrm{Fe}$ & $170[52,53]$ & 1530 & 190 \\
$\mathrm{~V}$ & $220[54]$ & 1580 & 235 \\
$\mathrm{Mo}$ & $472[55]$ & 3270 & 448 \\
$\mathrm{Ta}$ & $231[55]$ & 1740 & 252 \\
$\mathrm{Nb}$ & $232[55]$ & 1540 & 254 \\
$\mathrm{~W}$ & $790[56]$ & 4530 & 720 \\
$\mathrm{Fe}-25$ at.\%Ni & $398[6]$ & 3570 & 377 \\
$\mathrm{Fe}-3$ at.\%V & $90[57]$ & 1470 & 109 \\
$\mathrm{Fe}-3 a t . \% \mathrm{Si}$ & $298[10]$ & 4040 & 271 \\
\hline
\end{tabular}

double-layer fault $2 \gamma_{t s f}$ is formed, the dislocations have to overcome an additional fault energy represented by the $\gamma_{T B M}$ to form a twin. The higher the $\gamma_{T B M}$, the higher is the stress required to nucleate a twin. Equation (5) clearly shows the dependence of twinning stress on the GPFE and other important material parameters. In addition, when solute atoms are added to the crystal, there is a local distortion in the arrangement of atoms. For example, in case of $\mathrm{Fe}-\mathrm{Cr}$, the increase in Peierls stress with increasing $\mathrm{Cr}$ content and hence, the twinning stress has been validated by atomistic simulations [58] and recent single-crystal measurements [58].

A great wealth of experimental data suggest that critical twinning stress in bcc metals and alloys does not vary with deformation temperature $[6,10,52,53,59,60]$ in the range 4-300 K. As suggested by Kroupa and Vítek [61], the applied stress on fractional dislocations overcome the energy barriers to form a twin without the aid of thermal activation, resulting in twinning stresses that are nearly independent of temperature. 
Table 4. The separation distance between the fractional dislocations $\left(d_{1}\right.$ and $\left.d_{2}\right)$, the $\left\{\begin{array}{lll}1 & 1 & 2\end{array}\right\}$

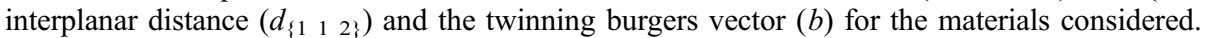

\begin{tabular}{lcccc}
\hline Material & $d_{1}(\AA)$ & $d_{2}(\AA)$ & $d_{\{112\}}(\AA)$ & $b(\AA)$ \\
\hline $\mathrm{Fe}$ & 88.88 & 147.97 & 1.16 & 0.823 \\
$\mathrm{~V}$ & 54.23 & 223.15 & 1.23 & 0.867 \\
$\mathrm{Mo}$ & 0.13 & 505.11 & 1.28 & 0.904 \\
$\mathrm{Ta}$ & 2.45 & 347.80 & 1.33 & 0.942 \\
$\mathrm{Nb}$ & 8.72 & 333.13 & 1.35 & 0.955 \\
$\mathrm{~W}$ & 0.13 & 560.99 & 1.29 & 0.909 \\
$\mathrm{Fe}-25$ at.\%Ni & 1.44 & 164.91 & 1.17 & 0.827 \\
$\mathrm{Fe}-3$ at.\%V & 87.78 & 27.06 & 1.18 & 0.832 \\
$\mathrm{Fe}-3 a t . \% \mathrm{Si}$ & 0.317 & 75.31 & 1.17 & 0.824 \\
\hline
\end{tabular}

Therefore, the twinning stress predictions reported in Table 3 are applicable to room temperature as well. A further analysis can be made on the competition between twinning and slip in the alloys considered in the present study by comparing the energy barriers associated with the nucleation of the first-layer stacking fault $\gamma_{u s}$ of a twin (see Table 2) to that of a full-slip dislocation $\gamma_{u s}^{\text {slip }}$. The unstable fault energies for slip $\left(\gamma_{u s}^{\text {slip }}\right)$ were obtained for $\mathrm{Fe}\left(710 \mathrm{~mJ} \mathrm{~m}^{-2}\right)$, Mo $\left(1310 \mathrm{~mJ} \mathrm{~m}^{-2}\right)$, V $\left(680 \mathrm{~mJ} \mathrm{~m}^{-2}\right), \mathrm{Nb}$ $\left(710 \mathrm{~mJ} \mathrm{~m}^{-2}\right)$, Ta $\left(690 \mathrm{~mJ} \mathrm{~m}^{-2}\right)$, W (1660 $\left.\mathrm{mJ} \mathrm{m}^{-2}\right)$ Fe-3at.\%Si $\left(30 \mathrm{~mJ} \mathrm{~m}^{-2}\right)$, $\mathrm{Fe}-25$ at.\%Ni $\left(570 \mathrm{~mJ} \mathrm{~m}^{-2}\right)$ and $\mathrm{Fe}-3 \mathrm{at} . \% \mathrm{~V}\left(701 \mathrm{~mJ} \mathrm{~m}^{-2}\right)$. We computed the ratio of $\gamma_{T B M}$ to $\Delta \gamma_{u s}\left(=\gamma_{u s}^{\text {slip }}-\gamma_{u s}\right)$ for these materials, and observed that the ratio of $\gamma_{T B M}$ to $\Delta \gamma_{u s}$ is much less than 1. From this observation, we conclude that the energy expense for nucleating a first-layer twin and successive faults is much less than the energy required to nucleate a slip. The reason is that twinning requires the emission of subsequent $b_{p}=a / 6\left\langle\begin{array}{lll}1 & 1 & 1\end{array}\right\rangle$ fractional dislocations on consecutive $\left\{\begin{array}{lll}1 & 1 & 2\end{array}\right\}$ planes. When under stress, the fractional dislocation $b_{p}=a / 6\left\langle\begin{array}{lll}1 & 1 & 1\rangle\end{array}\right)$ is formed, the competition between emitting a second fractional dislocation or continuing for a full-slip dislocation $a / 2\left\langle\begin{array}{lll}1 & 1 & 1\end{array}\right\rangle$ depends on the energy barrier to emit an $a / 3\left\langle\begin{array}{lll}1 & 1 & 1\end{array}\right\rangle$ dislocation on the same $\left\{\begin{array}{lll}1 & 1 & 2\end{array}\right\}$ plane (represented by $\gamma_{u s}$ ) or the energy to emit a subsequent $b_{p}=a / 6\left\langle\begin{array}{lll}1 & 1 & 1\end{array}\right\rangle$ dislocations (represented by $\gamma_{T B M}$ ) on consecutive $\left\{\begin{array}{lll}1 & 1 & 2\end{array}\right\}$ planes [62].

We note that the energy minima do not correspond to the same displacement fraction in all cases (see Figure 2) because of the type of the twin boundary. It is well known that two distinct $\left\{\begin{array}{lll}1 & 1 & 2\end{array}\right\}$ coherent twin boundaries exist in bcc crystals, namely reflective and isosceles [63]. The preference of one boundary over the other remains to be a topic of continuing interest [63-65]. In the present work, both boundaries have been considered in our calculations. In fact, we find that the energy difference between the two cases to be small (for example, for Mo and W, we calculate twinning stresses of 440 and $725 \mathrm{MPa}$, respectively, for the isosceles case which are within 5\% of the reflective twin case).

To conclude, we modified the original PN formulation to account for the twinning process in a wide range of bcc metals and alloys. The discrete form of the misfit energy integral in the original PN formalism was employed to accurately obtain the energy variation with respect to the position of the fractional dislocations forming the twin, hence much lower twinning stress was obtained compared to other models.

For the first time, we provide the theoretical and experimental results in a normalized plot that can be readily utilized to assess the twin stress in bcc metals and 
alloys (Figure 3). The relationship between the critical shear stress and the energy parameters is linear in a log-linear plot. We reveal several important parameters - (i) the total separation distance of the twinning fractionals, the interplanar spacing, the shear moduli and burgers vector, and (ii) the twin boundary migration energy, the unstable twin energy and stacking fault energy. This normalized twinning stress plot was insightful in pinpointing the major variables affecting the twinning stress dependence. One advantage of the proposed theory is that the twinning stress calculation shows excellent agreement with the experiments and requires no empirical constants.

\section{Acknowledgements}

The work was supported by the National Science Foundation, NSF CMMI-113003. This support is gratefully acknowledged.

\section{References}

[1] J.W. Christian and S. Mahajan, Prog. Mater. Sci. 39 (1995) p.1.

[2] S. Mahajan, Philos. Mag. 26 (1972) p.161.

[3] A.W. Sleeswyk, Philos. Mag. 8 (1963) p.1467.

[4] K.P.D. Lagerlöf, Acta Metall. Mater 41 (1993) p.2143.

[5] S. Mahajan, Acta Metall. 23 (1975) p.671.

[6] J.L. Nilles and W.S. Owen, The Society of Metals, Japan 97 (1972) p.97.

[7] R. Priestner and W.C. Leslie, Philos. Mag. 11 (1965) p.895.

[8] A.H. Cottrell and B.A. Bilby, Philos. Mag. Ser. 7(42) (1951) p.573.

[9] K. Ogawa, Philos. Mag. 11 (1965) p.217.

[10] M.A. Meyers, O. Vöhringer and V.A. Lubarda, Acta Mater. 49 (2001) p.4025.

[11] S. Ogata, J. Li and S. Yip, Phys. Rev. B 71 (2005) p.224102.

[12] P. Carrez, D. Ferré and P. Cordier, Philos. Mag. 87 (2007) p.3229.

[13] J. Hartford, B. von Sydow, G. Wahnström and B. Lundqvist, Phys. Rev. B 58 (1998) p.2487.

[14] B. Joós and M.S. Duesbery, Phys. Rev. Lett. 78 (1997) p.266.

[15] B. Joós, Q. Ren and M.S. Duesbery, Phys. Rev. B 50 (1994) p.5890.

[16] L. Lejček, Czech. J. Phys. 23 (1973) p.176.

[17] A. Ngan, J. Mech. Phys. Solids 45 (1997) p.903.

[18] A. Ostapovets and V. Paidar, Phys. Met. Metall. 111 (2011) p.229.

[19] Q. Ren, B. Joós and M.S. Duesbery, Phys. Rev. B 52 (1995) p.13223.

[20] G. Schoeck, Phys. Rev. Lett. 82 (1999) p.2310.

[21] G. Schoeck, Phys. Status Solidi B 248 (2011) p.2284.

[22] G. Schoeck and W. Püschl, Mater. Sci. Eng. A 189 (1994) p.61.

[23] E.B. Tadmor and S. Hai, J. Mech. Phys. Solids 51 (2003) p.765.

[24] V. Vitek and M. Yamaguchi, J. Phys. F: Met. Phys. 3 (1973) p.537.

[25] F.R.N. Nabarro, Proc. Phys. Soc. 59 (1947) p.256.

[26] E.B. Tadmor and R.E. Miller, Modeling Materials: Continuum, Atomistic and Multiscale Techniques, Cambridge University Press, Cambridge, 2011.

[27] A.J. Foreman, M.A. Jaswon and J.K. Wood, Proc. Phys. Soc., Sect. A 64 (1951) p.156.

[28] Y.-M. Juan and E. Kaxiras, Philos. Mag. A 74 (1996) p.1367.

[29] V. Vítek, Philos. Mag. 18 (1968) p.773.

[30] J. Wang and H. Sehitoglu, Acta Mater. 61 (2013) p.6790.

[31] J. Wang, H. Sehitoglu and H.J. Maier, Int. J. Plast. 39 (2012) p.61. 
[32] V. Vitek, Philos. Mag. 84 (2004) p.415.

[33] A. Ostapovets and V. Paidar, Mater. Sci. Forum 567-568 (2007) p.69.

[34] K. Ogawa and R. Maddin, Acta Metall. 12 (1964) p.713.

[35] G. Kresse and J. Furthmuller, Phys. Rev. B 54 (1996) p.11169.

[36] G. Kresse and J. Hafner, Phys. Rev. B 48 (1993) p.13115.

[37] D.E. Jiang and E.A. Carter, Phys. Rev. B 70 (2004) p.064102.

[38] D.J. Dever, J. Appl. Phys. 43 (2003) p.3293.

[39] J.B. Adams and S.M. Foiles, Phys. Rev. B 41 (1990) p.3316.

[40] A. Magerl, B. Berre and G. Alefeld, Phys. Status Solidi A 36 (1976) p.161.

[41] D.I. Bolef, J. Appl. Phys. 32 (1961) p.100.

[42] A.R. Jani, N.E. Brener and J. Callaway, Phys. Rev. B 38 (1988) p.9425.

[43] G. Das, Thin Solid Films 12 (1972) p.305.

[44] M.P. Zach, K.H. Ng and R.M. Penner, Science 290 (2000) p.2120.

[45] M. Alouani, R.C. Albers and M. Methfessel, Phys. Rev. B 43 (1991) p.6500.

[46] W.P. Davey, Phys. Rev. 26 (1925) p.736.

[47] F.H. Featherston and J.R. Neighbours, Phys. Rev. 130 (1963) p.1324.

[48] H. Zhang, M.P.J. Punkkinen, B. Johansson, S. Hertzman and L. Vitos, Phys. Rev. B 81 (2010) p.184105.

[49] H. Suzuki, J. Phys. Soc. Jpn. 17 (1962) p.322.

[50] S. Kibey, J. Liu, D. Johnson and H. Sehitoglu, Appl. Phys. Lett. 89 (2006) p.191911.

[51] D. Finkenstadt and D.D. Johnson, Phys. Rev. B 73 (2006) p.024101.

[52] J. Harding, Proc. R. Soc. A: Math. Phys. Eng. Sci. 299 (1967) p.464.

[53] J. Harding, Mem. Sci. Rev. Met. 65 (1968) p.245.

[54] J.W. Edington and R.E. Smallman, Acta Metall. 13 (1965) p.765.

[55] P.J. Sherwood, F. Guiu, H.-C. Kim and P.L. Pratt, Can. J. Phys. 45 (1967) p.1075.

[56] C.Y. Chiem and W.S. Lee, Mater. Sci. Eng. A 187 (1994) p.43.

[57] H.G. Suzuki, M. Tanino and K. Aoki, Jpn. J. Appl. Phys. 5 (1966) p.879.

[58] D. Farkas, C.G. Schon, M.S.F. De Lima and H. Goldenstein, Acta Mater. 44 (1996) p.409.

[59] D. Lohe and O. Vohringer, Z. Metallkd. 77 (1986) p.557.

[60] N. Narita and J.I. Takamura, Disloc. Solids 9 (1992) p.135.

[61] F. Kroupa and V. Vítek, Can. J. Phys. 45 (1967) p.945.

[62] S. Li, X. Ding, J. Deng, T. Lookman and J. Li, Phys. Rev. B 82 (2010) p.205435.

[63] M. Mrovec, T. Ochs, C. Elsässer, V. Vitek, D. Nguyen-Manh and D.G. Pettifor, Z. Metallkd. 94 (2003) p.244.

[64] A.G. Marinopoulos, V. Vitek and A.E. Carlsson, Philos. Mag. A 72 (1995) p.1311.

[65] S. Tsurekawa, T. Tanaka and H. Yoshinaga, Mater. Sci. Eng. A 176 (1994) p.341. 